\title{
Filigrane
}

Écoutes psychothérapiques

\section{Libre vagabondage}

\section{Claude Spielmann}

Volume 16, numéro 2, automne 2007

Les hauts lieux et non-lieux du rêve II

URI : https://id.erudit.org/iderudit/016918ar

DOI : https://doi.org/10.7202/016918ar

Aller au sommaire du numéro

Éditeur(s)

Revue Santé mentale au Québec

ISSN

1192-1412 (imprimé)

1911-4656 (numérique)

Découvrir la revue

\section{Citer cet article}

Spielmann, C. (2007). Libre vagabondage. Filigrane, 16(2), 15-26.

https://doi.org/10.7202/016918ar

\section{Résumé de l'article}

Nulle démonstration dans ce texte ; un simple vagabondage orienté. La part subjective et fictionnelle, loin d'avoir été écartée, est le moteur de l'écriture afin d'être au plus près du rêve. La fantaisie de l'écrivant, autant que faire se peut, n’a pas été recherchée alors que, sans aucun doute, la " Phantasie ", comme dit la langue allemande, est présente. En opposant arbitrairement songe à rêve, il est proposé de voir dans l'instance du rêve le lieu où s'origine la vérité subjective et le sentiment d'existence qui, à l'horizon, ne peut que renvoyer à " l'inconnu ", au réel, à la mort. Le rêve, comme style de discours, en témoigne sans jamais pouvoir s'en expliquer. Mais, prendre acte de son témoignage, peut ouvrir la possibilité de la transmission. Quelques courts fragments cliniques, sans volonté d'interprétation, illustreront ces propos.
Ce document est protégé par la loi sur le droit d'auteur. L'utilisation des services d'Érudit (y compris la reproduction) est assujettie à sa politique d'utilisation que vous pouvez consulter en ligne.

https://apropos.erudit.org/fr/usagers/politique-dutilisation/ 


\title{
Libre vagabondage
}

\author{
claude spielmann
}

Nulle démonstration dans ce texte; un simple vagabondage orienté. La part subjective et fictionnelle, loin d'avoir été écartée, est le moteur de l'écriture afin d'être au plus près du rêve. La fantaisie de l'écrivant, autant que faire se peut, n'a pas été recherchée alors que, sans aucun doute, la «Phantasie», comme dit la langue allemande, est présente. En opposant arbitrairement songe à rêve, il est proposé de voir dans l'instance du rêve le lieu où s'origine la vérité subjective et le sentiment d'existence qui, à l'horizon, ne peut que renvoyer à «l'inconnu», au réel, à la mort. Le rêve, comme style de discours, en témoigne sans jamais pouvoir s'en expliquer. Mais, prendre acte de son témoignage, peut ouvrir la possibilité de la transmission. Quelques courts fragments cliniques, sans volonté d'interprétation, illustreront ces propos.

a nuit qui a suivi ma décision d'écrire cet article, j'ai rêvé que, seul dans mon lit, j'étais fermement ligoté, sans pouvoir me défaire de mes liens. Curieuse rencontre entre le factuel et l'atemporel.

La vie est un songe, pièce de Calderon publiée en 1635. Le héros, Sigismond, a peut-être intéressé Sigismond Freud, mais je ne saurais dire s'il l'a connu. Je ne raconterai pas cette pièce dont le tumulte de pulsion, de rêve et de vérité mérite pourtant une lecture, comme, à son sujet, l'article court mais pertinent et amusant de Pierre Daviot, «La vie est un songe», paru en 2005 dans «Actualité de «L'interprétation du rêve » », Les carnets de psychanalyse, à la suite d'un colloque de l'InterAssociatif Européen de Psychanalyse. Je me suis moins intéressé à la pièce qu'à son titre qui a dû relâcher les liens de mon rêve. Ce titre et ce rêve ont, sans nul doute, rendu possible l'écriture de cet article. Mais auparavant, à propos du songe, quelques définitions du Littré que je ne manquerai pas d'oublier en cours d'écriture:

1. «Opérations irrationnelles des facultés intellectuelles en partie éveillées chez une personne qui dort. »

2. «Fictions comparées à un songe auquel on se livre tout éveillé.»

3. «Ce qui occupe l'esprit, sans avoir une réalité assurée.»

«J'ai tant rêvé de toi que tu perds ta réalité.» (R. Desnos)

Songer à parler de ses rêves. Deux termes, songe et rêve, souvent confondus. Le premier, peut-être plus désuet ou nostalgique. Songe ou rêve? Mais si les songes sont des mensonges...

Depuis quelques minutes, Benjamin regardait le ciel. De longues minutes. Regard noyé dans un bleu qu'aucun nuage ne troublait. Rien pour arrêter ni même accrocher le regard. Benjamin en était désemparé, attristé, presque égaré. Le ciel est sans limite. Vide? Trop plein, trop saturé ? Benjamin n'éprouvait pas de sentiment de solitude. Au fond, pensait-il, le bleu du ciel est comme le sans couleur de la nuit. 
Qui sait ce qu'est un rêve? Tout le monde, puisque tout le monde rêve. Mensonge, ment-songe diraient certains.

Le rêve dit-il la vérité et le songe ment-il ? Que signifie «la vie est un songe»? La vie serait un mensonge et le rêve la vérité ? Peut-être y a-t-il, en effet, curieusement, quelque vérité à dire les choses ainsi. Quelque vérité, simplement, peut parfois s'entendre au détour d'un vague récit quand s'insère une fausse note, c'est-à-dire la note juste, qui rompt la monotonie et l'ennui de l'écoute. Quand la silhouette de vérité entame l'inertie d'un discours qui tente de se constituer comme tel...

En fait, il vaudrait mieux dire: la vie a commerce avec du mensonge et le rêve avec de la vérité. Nuance de taille qui ne facilite pas la tâche. Brouille-piste apparent et, en réalité tentative d'un meilleur repérage. Ne redoutons pas l'arbitraire, l'approximation, la fiction, le détournement de sens, d'un sens qui se voudrait fixé: le songe errant est de l'ordre de l'imaginaire qui n'a pas trouvé de lieu; le rêve, en fondant de facto son lieu, est de l'ordre du sens. Le songe s'inscrit dans le temps vectorisé mais s'égare en chemin et nous perd; le rêve, toujours rappel fidèle, parcourt le temps qui nous traverse, nous affecte et nous porte plus avant. Le songe pose des lapins. Il est aussi fou que le «lièvre de mars » du Pays des Merveilles d'Alice (Carrol, 1990, 140). Alors qu'à notre insu, nous sommes engagés dans le rêve, passeur de sens, traducteur de sens; cet insu qui témoigne de notre engagement dans l'existence. Sagesse invisible du rêve.

Benjamin : Sarah est donc morte. Voila déjà quelques années. Sa mort a suivi la mort d'un autre et a précédé la mort d'une autre. De peu, pour l'un et l'autre. Trois vies essentielles, trois morts non moins essentielles. Sarah est le nom de ces trois morts qui ont constitué ma vie selon des modes et des temporalités différentes. L'une des trois a pourtant une place à part.

Or, parfois, je me demande si Sarah a vraiment vécu. Je veux dire vécu à mes côtés, avec moi, si nous avons construit nos vies ensemble. Je sais que oui, je le sais de mon savoir. Mais au-delà? Aujourd'hui, ma douleur naît de ce que ce savoir ne recouvre pas la conviction intime, l'absolue évidence. Était-ce donc un songe? Non. Oui. Non pendant, oui après?

Qu'y puis-je si, dans mes rêves seulement, que je rêve de Sarah ou non, je suis habité par cette conviction intime, celle de son existence avec moi, de notre histoire commune, de sa présence constitutive de ma propre histoire. J'ai la conviction intime que mes rêves disent quelque chose de ma vérité. Ils expriment ma vie en images et ce que je suis en images qui sont plus que des images. Pourtant, dès que je veux en parler, je sens que j'échoue, sachant pourtant que ces images sont des mots dissimulés. Et même, à dire ce que je dis maintenant, j'échoue. Je me manque. Je manque à moi-même. Je vous manque.

Tant pis. J'accepte. Même maladroitement, on ne peut pas ne pas dire ses rêves. D'ailleurs ce n'est que lorsqu'on tente de les dire qu'ils deviennent plus que des rêves. Tant que je rêve, je rêve. Donc je dors. Mais dès que je ne dors plus, je ne 
rêve plus et cependant, le rêve est toujours là. Situation étrange car s'il s'agit de deux états différents, où est l'intervalle entre les deux? Pas le fossé mais le passage? Éveillé, je retrouve quelque chose de mon sommeil déjà présent avant que je ne m'endorme et ne me mette à rêver.

Sarah morte, j'ai souvent rêvé d'elle. Mes rêves sortent des coulisses, lieux que le spectateur ne voit pas et que le comédien oublie en scène. Je ne suis pas un simple spectateur. Je le suis certes, mais suis aussi décorateur, acteur, machiniste et éclairagiste. Je rêvais à la souffrance obligatoire que suscite la présence d'un autre et à toute la haine que j'ai pu lui porter. La haine de t'aimer. Et je me demandais comment me libérer de ces liens amour-haine.

Je n'ai pas manqué d'y réfléchir et j'ai fini par m'autoriser à laisser ma pensée voyager où elle voulait. L'apaisement ne venait pas. Au contraire, les rêves s'enchaînaient.

Aujourd'hui, le calme, sinon la paix, m'habite, peut-être plus qu'il ne l'a jamais fait. Et j'ai retrouvé pour un temps des rêves plus ordinaires. Enfin parfois, des rêves de la vie qui est toujours quotidienne. Il n'y a pas d'éternité dans le quotidien. Mais au fond, le calme ne me va pas bien. Dieu merci, ce n'est pas la paix. Ce n'est pas la paix car je n'en ai pas fini avec vous, Sarah, mes trois morts. Vivants, vous avez été ce qui a compté le plus et ce qui m'a le plus heureusement dérangé. Je vous ai aimé vivants, et aujourd'hui ? Morts, je vous en veux.!

Benjamin: Je n'ai pas été à Auschwitz. À l'époque, j'aurais pu. J'avais l'âge. Mon père ni ma mère n'y sont allés. Mes oncles y ont été emmenés ainsi qu'une tante et deux cousines. Après, je ne suis jamais allé voir si Auschwitz avait existé. J'en ai tellement rêvé, j'ai tellement rêvé à tout ça, de près ou de loin, que je sais, d'un savoir qui ne peut se dire, qu'Auschwitz est inoubliable même pour ceux qui ne l'ont pas connu dans leur corps. J'ai lu, j'ai vu les images, le Film, les témoignages, les expositions qui exposent la déportation. Tout ça est important, évidemment. Mais la seule réalité pour moi, ce que je considère comme réalité, ce qui en moi, dans mon corps, est réalité, ce sont mes rêves. J'en ai tant rêvé.

Comme cette jeune femme née après la guerre, vivant dans un pays où la déportation n'avait pas sévi, dont aucun membre de la famille n'avait été victime, et qui ne cessait pourtant d'y rêver.

Je ne suis jamais allé à Auschwitz, ni pendant ni après. Pourtant, j'ai le sentiment de ne l'avoir jamais quitté. Ou plutôt, Auschwitz ne m'a plus quitté, même si je n'y pense pas. Alors, si la vie est un songe, le rêve n'est-il pas la vie même? Vie intime, vie intouchable. Celle qui ne peut que se danser.

Sarah, tu es intouchable, vous êtes mon intime intouchable. Je vous rêve par delà la mort et ma vie qui continue. Et je danse dans vos regards, je le sais.

«Non, mais je rêve!» Curieuse expression pour dire: ce que je prenais pour vrai est mensonge alors que le vrai est dans le rêve. La vérité que tu me révèles est celle-là même que me révèlent mes rêves. Ce que tu dis de moi m'a déjà été dit 
dans mes nuits. Éveillé, je n'en crois pas mes oreilles. Vérité de la nuit, vérité du jour. La même, en un langage différent. Les images du jour sont-elles plus incroyables que celles de la nuit? Vous, mes Sarah aveugles, sauriez-vous me dire si votre cécité absolue qui ne fait plus la différence entre le jour et la nuit, distingue, en dépit de tout, l'admissible de l'inadmissible? Ne me dites pas que j'écris à des morts puisque souvent, en rêve, vous me dites le contraire.

Parfois j'ai le sentiment que mes rêves s'amusent à me perdre, et parfois la conviction qu'ils m'orientent. Ils m'invitent plutôt à choisir un itinéraire nouveau si je veux bien faire un effort de déchiffrage. Mais il m'arrive de céder à la tentation d'errer dans leur étrange géométrie. De m'y complaire. Et je pourrais alors aller jusqu'à dire : regardez-moi, plaignez-moi, si la honte d'en jouir ne me saisissait pas. Mais quand j'entends qu'un rêve parle ma langue originelle, je me sens vivant.

Benjamin: Souvent, mes rêves mettent en scène celui qui sera ma vie lorsque j'aurai perdu la mienne, ma vie qui continue de devenir. C'est pour ça que je peux écrire. Sans doute, est-ce à lui que j'écris. Ce n'est pas moi qui le mets en scène, Je s'en charge à sa guise.

De quoi puis-je rêver lorsque je rêve d'un enfant, un enfant vivant?

Réceptacles, creusets de ma vie et de tout ce que je suis, mes rêves ne mentent pas. Dans leurs images condensées et déplacées, je sais qu'ils ne s'embarrassent ni de ratures, ni d'ajouts dans les marges ou de didascalies.

Mais alors, comment ne pas faillir à ma vie éveillée?

Claire dit: «Mes rêves sont plats. Si parfois j’y vois Victor, cet enfant, mort bien trop jeune, je ne ressens rien de ce que j'ai vécu avec lui. Rêves vides d'affects. Il me faut à tout prix retrouver, au moins dans ces images nocturnes, les sensations éprouvées avec lui, de son vivant, puisqu'il est mort. Continuer de ne voir que des images en à plat, images où manquent la chair et la troisième dimension me donne le sentiment qu'il n'a pas vraiment existé. Lui ai-je donné naissance? Me serais-je trompée ? Illusionnée ? Abusée ? Quelques années de vie avec lui où les mots échangés étaient ceux de la tendresse et de l'amour n'ont-ils été que ceux d'un songe mensonger?»

Claire aborde un rivage dangereux pour elle. Elle pensait qu'au moins dans ses rêves son enfant serait vivant. Or il ne l'est pas. Pire, elle sait bien qu'elle est sur une redoutable crête. Sur l'un des bords, la désillusion complète, sur l'autre la confirmation de l'amour fou, l'amour dans la folie car elle a été folle de son fils. Si cette part-là de sa vie est un songe, alors elle est passée à côté de toute sa vie. Si le rêve dit quelque chose sur le vrai, alors elle est aussi passée à côté puisque le rêve manque.

Sarah, j'ai tant rêvé de vous. Sans cesse j'ai tenté de conjoindre songe et rêve. Y suis-je parvenu? Je ne sais pas. Mais je suis toujours dans la vie, même si c'est 
avec quelque douleur. Je pense être davantage dans le rêve que dans le songe. Quelle que soit ma difficulté à en prendre acte, le rêve est mon allié. Je ne sens pas toujours ta chair ou ton odeur dans mes images de la nuit, mais souvent, la nuit, je sais, je pressens, je sens, j'ai la conviction de ne pas m'être planté dans ma putain d'existence. Mes Sarah, mon épaisseur, ma consistance, ma complétude. Et je ne suis pas passé trop loin de la place que vous m'avez offerte.

À quoi rêve-t-on lorsqu'on rêve d'un enfant?

Parfois dans mes nuits, surgit une phrase énigmatique que l'aube n'efface pas. Une sorte de formule, un brouillard de mots se présentant comme un condensé qui me laisse en suspend. Si la vérité ne peut que se mi-dire - à chacun de se laisser porter par elle —, elle est en peine de développement dans le rêve — à chacun alors de la déployer. Les phrases surgissant dans leur mystère, comme celle-ci par exemple : «il fallait que ce soit un enfant de la guerre», ne peuvent rester en l'état. L'urgence pousse à les dérouler et à les prolonger dans tous leurs possibles. D'ailleurs une phrase de rêve économise la moitié du travail, cette image de mots est déjà du mot, de la pensée en mots.

À quoi rêve-t-on lorsqu'on rêve d'un enfant?

L'un dit: «Je suis un handicapé de l'enfance.»

Un autre dit: «Je fais des malaises de vérité.»

Quel rapport peut-il y avoir entre ces deux phrases, s'il doit y en avoir un?

«Dit vrai qui dit les ombres» (P. Celan).

La vérité n'est pas stagnante au fond d'un puits. Et si elle est nue, elle gît nerveuse et s'agite, inaccessible depuis le premier matin. Déjà, elle ne reposait pas en paix puisqu'elle affrontait la dangerosité nécessaire de l'altérité qui se préparait.

La réalisation d'un désir infantile. D'un désir ou du désir, de l'instance du désir? Qu'est-ce qu'une instance? Première définition du Littré : «soin extrême, pressant». Il faut en effet un «soin extrême» pour ne pas se laisser détruire par l'autre, un «soin extrême» pour que l'autre devienne un allié avec qui se construire, un allié à qui s'adresser, adresser sa parole et ses rêves. Et puis, il est toujours «pressant» de s'adresser à cet autre multiple ou unique car il est «pressant» d'exister. Die Not des Lebens. Existence d'abord dépendante du soin de cet autre, exigeante aussi. Comment être dans la certitude d'une existence s'accomplissant, être dans la vérité qui conjoint la demande et le sentiment d'exister. Peut-être, d'ailleurs, est-ce dans la demande pressante que se dessine ce sentiment d'existence, lorsque cette demande n'est pas rejetée mais au contraire accueillie. L'accueil rassure, construit, autorise la demande à se développer pour devenir désir. Est-ce le désir qui pousse à la demande? Ne serait-ce pas aussi la demande qui donne visage au désir?

«... cette demande a une autre finalité, qui serait $[\ldots]$ de produire un manque chez l'autre [...] et, du même mouvement, de 
perpétuer ce manque même chez le sujet. [...] La fonction [du désir] ne serait pas d'obtenir la satisfaction, mais [...] de se préserver comme manque: c'est-à-dire d'arrimer le sujet à l'ordre de la demande » (Safouan, 2005, 160).

«Le rêve viserait [...] la permanence, le suspend du désir, non la satisfaction accomplie; l'objet du désir serait ici le désir même, alors que l'objet du désir de dormir est l'absolu, le point zéro de l'assouvissement» (Pontalis, 1972, 269).

Pourtant, la vérité est nue, pas encore revêtue des vêtements du semblant ou des stratégies qui apprivoisent l'autre. Le rêve est nu. Sa nudité impudique convoque ce sentiment d'existence grâce à l'érotique qui donne sa valeur, sa valeur, il faut insister sur ce mot, qui authentifie le sentiment. On ne peut sans doute pas en rendre compte mais le doute n'est pas possible. Cela est, rien d'autre à en dire. Traces où l'angoisse des premiers temps s'est inscrite, traces fondatrices qui exigent l'oubli et qui tentent de se frayer un chemin vers la conscience par des voies détournées, transformées, transfigurées, méconnaissables, brumes de Stimmung, bords insaisissables de certitude. Chacun établit un rapport transférentiel à son rêve, le rêve comme lieu supposé savoir.

«[...] entre les pensées du rêve et le rêve lui-même, une "transvaluation totale de toutes les valeurs psychiques"; la déformation n'a pu se faire que grâce à une diminution de valeur [...]» (Freud, 1900).

«Lacan précise: le rêve est désir, désir de dormir, entendez de ne pas se réveiller (pas seulement du sommeil). Et si l'on s'éveille dans l'angoisse ? C'est pour continuer de se protéger du réel» (Chemama, 1993, 252).

Et Lacan ajoute, cité par R. Chemama : «Le sujet du Wunsch se satisfait de l'être: organiser dans sa fuite métonymique l'être qu'appelle le langage, telle est selon nous l'essence du désir. » (Lacan, 1958-1959).

Voici peu, une femme me disait: «J'ai rêvé de cette image qui était en réalité une phrase : à part moi mais à part lui.» Peut-être faudrait-il l'écrire: à part moi, mais, à part lui. Ou bien: à part moi... mais... à part lui. La ponctuation est ici fondamentale. Elle est un élément structurel du rêve avant que le rêveur puisse se laisser porter par ses potentialités évocatrices. Elle n'en a pas dit plus. Je n'ai pas fait de commentaire et pourtant, depuis, sa phrase ne quitte pas mon esprit: à part moi, mais, à part lui. Fragments essoufflés, le souffle manque pour en dire 
davantage, pour mettre à la place des virgules le corps même de la pensée nocturne, le corps forcément impudique. Phrase où les virgules dissimulent, justement, l'impudeur. Mais, accepter l'autre ponctuation : à part moi... mais... à part lui, suscite une nuance. Moins violente, la phrase oppose une moindre défense, elle est même plus invitante. L'essoufflement disparaît au profit d'un flux régulier et poétique, d'une douceur séduisante. Elle reflète une tension qui déborde l'énoncé. Les points de suspension ne sont pas des scansions, contrairement aux virgules. Les points de suspension, dans cette phrase, dessinent la place d'un sujet en peine de mots et néanmoins, sur les rives du symbolique. Ils représentent les lieux où les mots manquent à dire, où les mots manqueront toujours pour que l'existence demeure en tension. Ils sont le souffle et non l'essoufflement. Ils sont la trace en transformation et en évolution d'une jouissance toute, fondatrice. Ils sont la nostalgie de cette trace et l'esquisse d'un désir adressé, alors qu'il ne peut s'exprimer.

Existe-t-il une ponctuation dans les rêves ? Il n'y a pas à en douter mais elle est invisible, variable et instable. Ne serait-elle pas ici particulièrement évocatrice, le «point de surgissement du rapport du sujet au symbolique» dont parle Lacan (1954-1955)?

Benjamin: J'ai rêvé qu'un homme me ceinturait de toutes ses forces. Derrière moi, il m'empêchait de faire le moindre mouvement. Je voulais crier mais malgré mes efforts, aucun son ne sortait de ma bouche. Finalement, j'ai pu émettre une sorte de râle où je n'ai pas reconnu le son de ma voix. J'ai éprouvé une détente complète tandis que le rêve s'arrêtait. J'ai su qu'il s'arrêtait alors que j'étais encore dans le rêve. J'ai senti la force de ce corps contre le mien. J'ai ressenti mes efforts pour m'en dégager. C'était effrayant et extraordinairement vivant. Dès avant mon réveil, sa valeur érotique était évidente. $\mathrm{Si}$, ensuite, j'ai pensé à l'homosexualité, cette idée s'avère insuffisante et même réductrice. C'est avant tout un rêve de corps. Mais de quel corps? Un corps entièrement corps - ou presque - encore muet de mots, un corps qui serait coupé du mien aujourd'hui, n'étaient ces traces troublantes qui troublent l'adulte exilé de son premier corps. Ce corps oublié, pourtant présent et inoubliable qui s'agite, infatigable, dans les méandres de la mémoire. Ce corps en proie aux forces pulsionnelles qui n'en peut plus de ne pas pouvoir, un corps-esprit emprisonné qui ne demande qu'à s'échapper, tout entier tendu vers un advenir et un devenir. Or, «les conditions de la formation des rêves » résident précisément dans ces «racines pulsionnelles», dit Freud. Si leurs modalités varient, les pulsions n’ont pas d'âge. Exubérantes ou assoupies, compagnes fidèles ou non, garant de notre sentiment d'existence, elles nous assurent que la vie, entre la non vie, existe.

Il n'est pas étonnant que la privation délibérée de rêve conduise à la folie.

Mais ce même corps devait éprouver la douceur des bras qui le rassuraient en le maintenant prisonnier, et en jouir. Alors, pourquoi l'image d'un homme dans ce rêve-là ? Et de quelle homosexualité s'agirait-il ? Au fond cela a-t-il une quelconque importance? Chacun sait qu'il conviendrait mieux de parler simplement de 
sexualité, et même de sexualité en cours. Il semble encore plus juste de dire: j'ai rêvé de l'époque où mon corps, de toujours sensible à la voix, devenait érogène.

«[...] le sommeil, du point de vue somatique, est une reviviscence du séjour dans le corps maternel...» (Freud, 1926, 126, cité par Fédida)

qui écrit encore :

«Comme si [...] l'homme jouait, chaque nuit, l'illusion de la perte en trouvant dans le rêve la satisfaction de son manque et la preuve donnée de sa propre immortalité » (Fédida, 1972, 229).

Et aussi :

«Le rêve [...] est paradoxalement le garant d'une réalité qui, en tant que réalité originaire, ne peut être décelée que par le désir et rester inscrite dans le corps même du fantasme» (Fédida, 1972, 229).

Madeleine, sur le divan, ponctuait régulièrement ses silences par: «Rien ne vient... je ne sais pas...», balbutié, à demi avalé. Ces quelques mots essentiels, murmures de sanglots, étaient, sans nul doute, le résultat d'un grand effort pour dire au moins: «Je suis là, ne m'oubliez pas.» Ils traduisaient des tentatives pathétiques pour retrouver les mots qui ne s'étaient pas inscrits, faute d'avoir été entendus au temps où ils auraient dû l'être.

Madeleine disait ne pas dormir et donc ne pas rêver.

Madeleine s'empêchait-elle de dormir pour ne pas rêver? Avait-elle si peur des rêves qui auraient pu surgir? Ou alors lui manquait-il les mots nécessaires pour rêver?

«Rien ne vient... je ne sais pas», a-t-elle répété tant de fois, comme une demande de mots qui, avant de pouvoir être dits, auraient été à entendre. Sans doute, faut-il des mots inscrits sur le corps pour que naissent des images exprimant les masques du désir. Et pourtant, elle n'était pas sans désir et se savait exister. Ce savoir s'exprimait dans ce «je ne sais pas... rien ne vient» qui traduisait en même temps «un désir de rien» (Lacan) auquel les rêves renvoient aussi. «Rien ne vient... je ne sais pas », esquisse d'une représentation impossible de la mort à laquelle ses parents avaient eu professionnellement affaire. Madeleine ne pleurait pas, mais des larmes discrètes, comme échappées, mouillaient presque tendrement ses joues. Elle me disait au revoir avec un regard désolé, en haussant légèrement les épaules comme lors d'une visite de condoléances.

Elle est ainsi restée prisonnière de sa vie comme d'un songe, jusqu'à ce qu'un rêve la surprenne dans un sommeil retrouvé. Premier rêve et premier sommeil ou première fois où ceux-ci pouvaient être reconnus et pris en compte? Dès lors, et d'autres rêves 
surgissant, le sentiment de son existence s'est trouvé quelque peu changé : l'espace du rêve s'est dégagé de celui du songe, une part du « désir de rien » s'est déplacé et repéré dans les rêves. Là où jusqu'alors «rien ne vient» était entièrement pris dans le désir de rien, cet X dont parle Lacan, cet innommable «apparenté à l'innommable par excellence, c'est-à-dire la mort» (Lacan, 1954-1955) est venu se glisser un espace où le désir commençait à ne plus être un simple mot courant. Madeleine met désormais en regard désir de rien et désir pour... et pose la question pourquoi.

Le rêve ne peut être réduit à son interprétation, il est mouvement psychique et se constitue en récit. Ça parle en nous. Le rêve est un style de discours.

«Le style est pour Lacan un opérateur à la jonction de la vérité de la cure et du savoir transmissible de cette vérité» (Porge, 2005, 45).

Certes, si une cure peut être émaillée de rêves, un rêve n'est pourtant pas une cure. Mais pour chaque rêveur, dès le réveil, il est nécessairement une mise en récit, un dire sur... de la vérité du sujet. Le propre du style est de pouvoir dire la même chose de manières différentes. Cette même Chose, das Ding, qui n'existe pas mais qui ne cesse de vouloir être retrouvée.

«Dans le trou de la Chose, viennent les choses qui disent la vérité » (Porge, 2005, 79).

Cette même chose qui, in fine, n'est autre que la vérité, celle dont parle Lacan lorsqu'il dit :

«Moi la vérité, Je parle» (Lacan, 1966, 409),

précisant plus tard:

«Nous revenons à proprement énoncer dans le Je ce qui donne le fond proprement de la vérité en tant qu'elle parle seulement » (Lacan J., 1968).

À qui parle-t-elle? À qui Je parle-t-il ? Ne serait-ce pas à Moi qui veut et ne veut rien en savoir?

À quoi rêve-t-on lorsqu' on rêve à un enfant vivant?

Ne serait-ce pas aussi à la non-vie qui encadre la vie?

Serait-ce à l'enfant, à l'infans en mouvement et en lutte pour en sortir (struggle for life), pour sortir de la non-vie et affronter sa mort? Cet enfant-là survit en chacun pour mener le même combat. 
«Les rêves [...] gardent souvent un point obscur [...]. C'est "l'ombilic" du rêve, le point où il se rattache à l'inconnu» (Freud, 1967).

L'X du rêve ne s'atteint pas, ne s'éteint pas. Articulé au désir pour..., il est la vérité nue, impudique, et produit chez le sujet la certitude d'une existence.

La certitude de l'existence ignore le temps. Tant que l'existence se déroule, elle cherche sa certitude. Elle ne se conjugue qu'au présent, et même au présent du singulier. Ce temps présent est celui, précisément, «où l'on représente le souhait comme accompli» (Freud, 1967). Et Roger Dadoun :

«Le rêve [...] est aussi force et vitalité, c'est-à-dire énergie libidinale, puissance sexuelle; et cette puissance, cette énergie de vie élémentaire, originaire — c'est cet "originaire" que désigne le concept de "temps du rêve", et c'est un originaire qui est cependant toujours présent, toujours actuel, comme l'activité onirique elle-même [...]» (Dadoun, 1972, 244).

Et, plus loin :

«[...] ne convient-il pas mieux de concevoir la sexualité comme onirique et d'avancer qu'une des fonctions vitales du rêve est de fabriquer la sexualité [...]?» (Dadoun, 1972, 252).

Le rêve parle vrai. Il parle du vrai. Le songe habille le vrai. Il trompe son monde. Il voile le vrai et par là même le dévoile partiellement. Il le rend sensible, désirable et redouté, imprévisible et presque étranger à soi. La vie est un songe.

Un matin, Sarah s'est réveillée d'un sommeil de cinq jours. Elle a parcouru des yeux le mur en face d'elle reconnaissant les tableaux à la même place. Elle avait à cet instant le regard clair et étonné d'un enfant. Ensuite, elle a refermé les paupières. Moment dérobé au sommeil, semblable à un rêve déchirant l'inconnu : Sarah se savait chez elle. Fausse note juste. Le rêve bref suffit à effleurer le vrai dans une fulgurance. Soupire au milieu d'une portée où s'entend le souffle de la mélodie. Regarder le rêve dans les yeux, saisir son regard qui plonge dans vos yeux. Comment s'y dérober?

Benjamin: J'ai rêvé d'une salle de spectacle. Je sais que Sarah est là mais je ne la vois pas. On me plante un poignard dans le dos, je ne saigne pas, je n'ai pas mal. Je ne trouve pas Sarah. Devant un comptoir, je dépasse une file de personnes. Un policier me laisse passer parce que je suis blessé. Puis, je voyage seul en train, sachant que Sarah s'y trouve. Défile un paysage de neige et d'eau. J'arrive dans 
une ville, le poignard toujours enfoncé dans mon dos. Je me perds. Je ne parviens pas à rejoindre Sarah.

Je sais aujourd'hui que même en elle, même lorsque sa jouissance et la mienne s'accordaient, je ne parvenais pas à la rejoindre dans l'intime de son corps. Je me plongeais dans le regard d'eau qui, alors, était le sien à cet instant et à d'autres. Elle m'y attirait et je me noyais dans l'abolition tendre de moi-même. Moment de nostalgie enfantine. Alors, je finissais par fermer les yeux.

Pourquoi ferme-t-on les yeux des morts? Leurs yeux sans regard sont-ils insupportables? Serait-ce la mort qui nous regarde? Les yeux des morts sont-ils des yeux avec trop de regard? Sont-ils l'impossible, le Réel qui nous aveugle? Est-ce là ce qui échoue à se représenter, même dans le rêve: l'ombilic, l'x. Les yeux des morts n'appartiennent ni au rêve ni au songe. Le rêve s'arrête au seuil de l'intraduisible, au bord de l'inerte, même lorsqu'il se nomme cauchemar. Il s'arrête à la limite où le désir pourrait s'annuler. Mais il ne s'annule pas. «Garant du sommeil » certes, garant aussi de l'existant, de l'existence et d'une vie possible.

La nuit m'est agréable. Je n'ai pas peur de m'endormir. Je m'attends toujours à visiter ce lieu familier et toujours inconnu qui, éveillé, me portera plus avant au moins jusqu'à la prochaine nuit. J'espère une nouvelle rencontre avec vous, Sarah. Elle ne sera peut-être pas heureuse, elle risque même d'être douloureuse. Mais si elle se produit, elle me conduira, à coup sûr, à la source originelle renouvelée car jamais tarie et constituera un pousse à vivre. Je le sais d'expérience et non de savoir.

Ainsi, de nuit effacée en nuit réussie, je trace sans doute un avenir qui ne m'échappera pas tout entier si j'ai le désir de le transmettre. Je déroule devant moi un temps dont j'aurai été éliminé mais dans lequel un enfant continuera de se débattre et de s'ébattre. À dire cela, je ne pense pas être dans le songe. Au contraire, le rêve comme garant trouverait là sa pleine fonction.

À quoi rêve-t-on lorsqu' on rêve à un enfant, à un enfant vivant?

La transmission est un rêve. Rêver et tenir compte de son rêve, c'est accepter le désir de transmettre, de transmettre à son insu quelques bribes de vérité qui échappent. Pour ne pas s'échapper.

Juillet 2006

Merci à Sophie Cathala-Pradal

claude spielmann

1 , rue boyer barret

75014 paris

claude.spielmann@wanadoo.fr 
Filigrane, automne 2007

\section{Références}

Carrol, L., 1865, Alice au pays des merveilles, Euvres, Paris, Gallimard, La Pléiade, 1990.

Chemama, R., Dictionnaire de la psychanalyse, Paris, Larousse, 1993.

Dadoun, R., Les ombilics du rêve, Nouvelle Revue de psychanalyse, ${ }^{\circ}$ 5, Paris, Gallimard, 1972..

Daviot, P., La vie est un songe, Actualité de «L'interprétation du rêve », Les carnets de psychanalyse, colloque de l'Inter-Associatif Européen de Psychanalyse, 2005.

Fédida, P., L'hypocondrie du rêve, Nouvelle Revue de psychanalyse, $\mathrm{n}^{\circ}$ 5, Paris, Gallimard, 1972.

Freud, S., 1900, L'interprétation des rêves, Paris, PUF, 1967.

Freud, S., 1926, Métapsychologie, Paris, Gallimard, 1940.

Lacan, J., 1954-1955, Séminaire II, Le Moi dans la théorie de Freud et dans la technique de la psychanalyse, Paris, Seuil, 1978.

Lacan J., 1955, La chose freudienne ou Sens du retour à Freud en psychanalyse, Écrits, Paris, Seuil, 1966.

Lacan, J., 1958-1959, Séminaire VI, Le désir et son interprétation, Paris, Seuil

Lacan, J., 1968, Séminaire VI, D’un Autre à l'autre, Paris, Seuil, 2006.

Pontalis, J.-B., La pénétration du rêve, Nouvelle Revue de psychanalyse, nº 5, Paris, Gallimard, 1972.

Porge, E., Transmettre la clinique psychanalytique, Ramonville Saint-Agne, Eres, Point Hors Ligne, 2005.

Safouan, M., La métaphore du rêve, Actualité de "L'interprétation du rêve », Les carnets de psychanalyse, colloque de l'Inter-Associatif Européen de Psychanalyse, 2005. 